\title{
Analysis of goalkeepers' game performances at the 2016 European Football Championships
}

\author{
A Kubayi, (iD DPhil (Sport Science) \\ Department of Sport, Rehabilitation and Dental Sciences, Faculty of Science, \\ Tshwane University of Technology, Pretoria, South Africa
}

Corresponding author: A Kubayi (kubayina@tut.ac.za)

Background: Despite a substantial body of literature on the physical and technical demands of outfield players in football, there is little information regarding the performance of goalkeepers.

Objective: The aim of this study was to analyse the game performance profiles of goalkeepers at the 2016 European Football Championships.

Methods: A total of 30 goalkeepers from 15 games played during the 2016 European Football Championships were analysed using the InStat ${ }^{\circledR}$ video tracking system.

Results: The results showed that goalkeepers covered a mean total distance of $4819 \mathrm{~m}$, ranging from $4036 \mathrm{~m}$ to $6640 \mathrm{~m}$. Overall, $68 \%$ of distance travelled was attributed to walking, whereas $0.8 \%$ was due to high-intensity activities. The goalkeepers of teams that lost matches covered significantly ( $p$ $<0.05$ ) longer distances while sprinting than those of teams that drew or won the matches. Goalkeepers of teams that drew significantly $(p<0.05)$ had a greater number of passes than those goalkeepers of teams that won or lost.

Conclusion: The current results have implications for soccer coaches to structure training sessions and tactical strategies for goalkeepers. The ability of goalkeepers to meet the physical and technical demands of a match could directly influence the successful execution of skills and the outcome of the competition.

Keywords: physical performance, distance, technical skills, matches

S Afr J Sports Med 2020;32:1-4. DOI: 10.17159/2078-516X/2020/v32i1a8283

The primary function of a soccer goalkeeper is to defend his/her goal, while the secondary role is to initiate attack through ball distribution. ${ }^{[1,2]}$ One of the greatest challenges a goalkeeper faces is that one mistake can cost the team its success. Almost every situation in which the goalkeeper is called into play is a high-pressure event, as it may potentially be a losing or winning situation in the match. ${ }^{[1]}$ Thus, a goalkeeper needs to possess a unique physical and technical profile, and it is likely that further details about their match-play and training demands would benefit practitioners seeking to optimise a training prescription for this playing population. ${ }^{[2]}$

Over the last decade, technological developments have seen the implementation of increasingly advanced video and motion analysis systems for collecting data on the physical and technical demands of soccer players. ${ }^{[3]}$ A number of studies has predominately focused on investigating the running demands of outfield players, ${ }^{[2-4]}$ with players covering total distances of 8-12 km during a soccer game. ${ }^{[5]}$ Conversely, limited research has analysed the physical demands of soccer goalkeepers. A review by White et al. ${ }^{[2]}$ demonstrated that goalkeepers cover total distances of four to six $\mathrm{km}$ during a match and appear not to experience between-half reductions in physical performance as the match progresses.

In addition to the reduced distances covered by goalkeepers, research has also found that the majority of this distance is covered at low-intensity levels, such as walking or jogging. ${ }^{[6]}$ With respect to comparisons between players, it has been found that goalkeepers performing at an international tournament spend approximately $98 \%$ of the game in the lowintensity threshold, compared to outfield players who spend approximately $83 \%$ of their time in this intensity zone. ${ }^{[7]}$ Furthermore, goalkeepers in the English Premier League were found to only spend $1 \%$ of their time in high-speed running (i.e. between 19.9 and $25.2 \mathrm{~km} / \mathrm{h}$ ), which was accounted for by approximately 10 high-speed runs and two sprints of less than 10 metres $(>25.2 \mathrm{~km} / \mathrm{h})$ per match. ${ }^{[6]}$ While goalkeepers may only perform two short sprints per match, these actions could represent important phases of play directly related to key situations that can influence the score and outcome of the match.[2]

Despite there being few studies on the physical demands of goalkeepers in soccer matches, ${ }^{2,6}$ there is little information on the technical performance of goalkeepers during match-play. It is essential to consider the technical aspects of the goalkeepers $[4,6]$ because this information can better predict successful team performance in soccer compared to purely physical parameters. ${ }^{[8]}$ Therefore, research on both physical and technical performances of goalkeepers could assist soccer coaches to better understand their specific match-related activities and training programmes can be modified appropriately. ${ }^{[3,4]}$ The purpose of this investigation was to analyse the game performance profiles of goalkeepers at the European Football Championships.

\section{Methods}

\section{Match sample and data collection}

A total of 30 goalkeepers from 15 games played during the 2016 European Football Championships were analysed using the InStat ${ }^{\circledR}$ video tracking system (https://instatsport.com/ football). Match activities were recorded using two video cameras installed on tripods set at the end of each stand. Video recordings from each camera fully covered separate halves of the field. The reliability of the InStat ${ }^{\circledR}$ tracking system has been demonstrated in previous research. ${ }^{[9]}$ Ethical clearance was received from the author's institutional ethics committee.

\section{Physical and technical indicators}

Physical indicators of goalkeepers were categorised as follows: (a) walking $(0-7 \mathrm{~km} / \mathrm{h})$, (b) jogging (7.1-14.5 km/h), (c) running $(14.6-20 \mathrm{~km} / \mathrm{h}),(\mathrm{d})$ high-speed running $(20.1-25 \mathrm{~km} / \mathrm{h})$ and (e) sprinting $(>25 \mathrm{~km} / \mathrm{h})$. High-intensity activity was defined as 
high-speed running and sprinting $(>20.1 \mathrm{~km} / \mathrm{h})$. Distances in attack and defence were calculated according to whether the goalkeeper was in possession of the ball or not. All distances in various categories were combined to calculate the total distance covered.

The technical indicators included variables such as save (the goalkeeper prevents the ball from entering the goal with any part of his body), pass (an intentional played ball from the goalkeeper to his teammate, including ball throwing from the hand), pass accuracy (\%) (a ratio calculated from successful passes divided by all passes), aerial duels won (\%) (two players competing for a ball in the air - for it to be an aerial duel, both players must jump and challenge each other in the air and have both feet off the ground), tackle (act of gaining possession from an opposition player who is in possession of the ball), lost ball (the goalkeeper lost ball possession due to a mistake/ poor control, including turnovers, dispossession, and unsuccessful passes), ball recovery (the event given at the start of a goalkeeper's recovery of ball possession from opponents from open play), foul drawn (where the goalkeeper is fouled by an opponent), and yellow-card (where the goalkeeper is booked by the referee due to illegal actions). ${ }^{[4,10]}$

\section{Statistical analysis}

Data were reported as means \pm standard deviations. Oneway analysis of variance was used to examine differences on the physical and technical parameters of goalkeepers based on match outcomes (i.e. win, lose or draw). In addition, if the $F$-ratio was significant at $p \leq 0.05$, then Tukey's HSD post hoc analysis was conducted. The effect size (ES) was used to determine the magnitudes of the studied variables. ES was grouped as follows: trivial $(<0.20)$, small $\quad(0.20-0.59)$, moderate (0.60-1.19), large (1.20-2.00), and very large (>2.00). ${ }^{[11]}$ All analyses were computed using the IBM Statistical Package for the Social Sciences (SPSS), version 25.0 . game period and phases of play $(n=30)$

$m$, metres

$m$, metres; $\%$, percentage; $n$, number

\section{Results}

Table 1 presents descriptive characteristics of distances covered at different intensities by goalkeepers. The mean total distance covered by goalkeepers was $4819 \mathrm{~m}$ (range $=4036 \mathrm{~m}$ to $6640 \mathrm{~m}$ ). Walking accounted for $68 \%$ of distance travelled during matches, with only $0.8 \%$ of distance covered spent in high-intensity activities, such as high-speed running and sprinting. There were minimal differences in the distances covered during the first half $(2412 \pm 281 \mathrm{~m})$ and second half $(2408 \pm 323 \mathrm{~m})$ of matches, with trivial effect $(\mathrm{ES}=0.01)$. Goalkeepers covered longer distances during attacking phases of play $(1660 \pm 463 \mathrm{~m})$, compared with defending $(1569 \pm 436$ m) phases $(E S=0.20$, small effect).

Table 1 . The mean distances $(\mathrm{m})$ covered by goalkeepers during a match relative to the different intensities,

\begin{tabular}{lcccc}
\hline & Mean & Standard Deviation & Minimum & Maximum \\
\hline Total distance (m) & 4819 & 580 & 4036 & 6640 \\
Walking & 3277 & 367 & 2590 & 3990 \\
Jogging & 1272 & 395 & 744 & 2127 \\
Running & 230 & 108 & 81 & 569 \\
High-speed running & 39 & 28 & 11 & 113 \\
Sprinting & 2.2 & 6.5 & 0 & 30 \\
First half & 2412 & 281 & 2048 & 3080 \\
Second half & 2408 & 323 & 1971 & 3560 \\
In defence & 1569 & 436 & 881 & 2645 \\
In attack & 1660 & 463 & 874 & 2767 \\
\hline
\end{tabular}

Table 2. Physical and technical indicators of goalkeepers according to the match outcome $(n=30)$

\begin{tabular}{lcccc}
\hline & Win & Lose & Draw & $\begin{array}{c}\text { Significance } \\
\text { (p-value) }\end{array}$ \\
\hline Physical indicators & & & & \\
$\quad$ Total distance (m) & $4808 \pm 433$ & $4787 \pm 771$ & $4880 \pm 522$ & 0.94 \\
Walking (m) & $3247 \pm 448$ & $3267 \pm 292$ & $3333 \pm 380$ & 0.88 \\
Jogging (m) & $1304 \pm 285$ & $1241 \pm 448$ & $1272 \pm 491$ & 0.94 \\
Running (m) & $231 \pm 83$ & $231 \pm 135$ & $226 \pm 112$ & 0.99 \\
High-speed running (m) & $27 \pm 16$ & $43 \pm 32$ & $49 \pm 31$ & 0.20 \\
Sprinting (m) & $0.0 \pm 0.0$ & $6.0 \pm 9.9^{*}$ & $0.0 \pm 0.0$ & $0.04^{*}$ \\
In defence (m) & $1480 \pm 423$ & $1432 \pm 354$ & $1880 \pm 447$ & 0.06 \\
In attack (m) & $1518 \pm 305$ & $1591 \pm 529$ & $1953 \pm 466$ & 0.10 \\
Technical indicators & & & & \\
Save (n) & $4.6 \pm 2.5$ & $3.8 \pm 1.7$ & $2.9 \pm 2.0$ & 0.21 \\
Pass (n) & $29 \pm 9$ & $28 \pm 8$ & $40 \pm 9^{*}$ & $0.01^{*}$ \\
Pass accuracy (\%) & $87 \pm 4$ & $88 \pm 9$ & $89 \pm 6$ & 0.83 \\
Aerial duel won (\%) & $64 \pm 51$ & $18 \pm 41$ & $13 \pm 35$ & 0.21 \\
Tackle (n) & $0.09 \pm 0.30$ & $0.18 \pm 0.40$ & $0 \pm 0$ & 0.45 \\
Lost ball (n) & $3.3 \pm 1.4$ & $2.9 \pm 2.8$ & $3.3 \pm 2.0$ & 0.90 \\
Ball recovery (n) & $5.6 \pm 2.0$ & $5.5 \pm 3.0$ & $6.6 \pm 4.0$ & 0.68 \\
Foul drawn (n) & $0.09 \pm 0.30$ & $0.18 \pm 0.40$ & $0.25 \pm 0.46$ & 0.68 \\
Yellow card (n) & $0.09 \pm 0.30$ & $0 \pm 0$ & $0 \pm 0$ & 0.44 \\
\hline Data are expressed as mean \pm standard deviation. ${ }^{*}$ Significant at $p<0.05 ;{ }^{*}$ Significantly higher than other teamsat $p<0.05$. \\
(n)
\end{tabular}


Table 2 shows distances covered by goalkeepers at different intensities, as well as technical indicators based on match outcome. Goalkeepers of teams that lost covered significantly greater distances while sprinting $(6.0 \pm 9.9 \mathrm{~m})$ compared with those of teams that drew or won games $(F[2,27]=3.48, p=$ 0.05). Post hoc comparisons showed that the mean score for a sprinting distance of goalkeepers of teams that lost was significantly higher than for teams that won or drew. Goalkeepers of teams that drew covered greater distances associated with walking $(3333 \pm 380 \mathrm{~m})$ and high-speed running $(49 \pm 31 \mathrm{~m})$. Goalkeepers of the teams that won covered longer distances when defending $(1480 \pm 422 \mathrm{~m})$, while those of teams that lost $(1591 \pm 529 \mathrm{~m})$ or drew $(1953 \pm 466 \mathrm{~m})$ covered greater distances associated with attacking phases.

With regard to the technical variables, a statistically significant difference was observed on passes $(F[2,27]=4.61$, $p=.01)$. The post hoc comparisons showed that the mean score of the passes for the goalkeepers of teams that drew $(40 \pm 9)$ was significantly different from those of teams that won (29 \pm $8, \mathrm{ES}=1.21$, large effect $)$ or lost $(28 \pm 8, \mathrm{ES}=1.45$, large effect $)$. Goalkeepers of teams that won had a higher number of aerial duels won, albeit not significant, than those of teams that lost $(\mathrm{ES}=1.71$, large effect).

\section{Discussion}

The aim of this study was to analyse the game performance profiles of goalkeepers at the 2016 European Football Championships. Regarding physical parameters, goalkeepers covered an average total distance of $4819 \mathrm{~m}$ during a match. This result is lower than that of previous findings, which reported that goalkeepers covered a total distance of $5611 \mathrm{~m}$ during match-play. ${ }^{[6]}$ The current study demonstrated that there are minimal differences between the halves of the game in terms of the distance covered by goalkeepers. This observation is consistent with that of Di Salvo et al.[6] who found that the physical performance for goalkeepers is mirrored across the two halves. Regardless of variations between studies, these distances represent about $50 \%$ of those covered by outfield players and may explain why no betweenhalf declines in total distance have been observed within any intensity threshold for international goalkeepers. ${ }^{[2]}$

As indicated in previous studies, ${ }^{[2,6]}$ goalkeepers walked for $68 \%$ of the total match duration and spent only $0.8 \%$ of the match in high-intensity activities. This finding suggests that as goalkeepers work in a limited space (i.e. in the defensive penalty area), it may be difficult for them to display a large number of high-intensity actions during a match. ${ }^{[6]}$ Despite their low high-intensity running distances, this may, however, represent actions which can have a direct influence on the outcome of a match, ${ }^{[2]}$ and therefore coaches should design training sessions involving high-intensity, game-specific activities. Regarding running performance and match outcome, the finding that the goalkeepers of the teams that lost covered significantly greater distances in sprinting compared to the goalkeepers of the teams which won or drew, could be explained by the fact that they may push forward when losing, thus reaching their maximal physical capacity in the hope of potentially drawing or winning the game. ${ }^{[12]}$

The present data further showed that goalkeepers of teams that drew covered a greater distance in the defending phases of play than those of the teams that lost or won. From a defensive perspective, this finding could be associated to the fact that teams that draw may adopt a more defensive strategy to ensure that they do not concede and potentially lose the game. In this context, players may adopt a ball retention strategy, ${ }^{[13]}$ which could result in higher distance covered when defending during a match. Maintaining possession of the ball is one of the most physically demanding playing styles because, if executed appropriately, players (including goalkeepers) may have to expend more energy during a match. ${ }^{[14]}$

Furthermore, the goalkeepers of teams that drew or won had a greater number of passes than those of teams that lost. In modern soccer, goalkeepers need to be proficient in their ball control skills, such as passing, so that without the option to use their hands, back-passes from teammates are secured to better deal with opponent pressure. ${ }^{[15]}$ Goalkeepers of teams that won had a higher number of successful aerial duels, with a large magnitude, compared to the goalkeepers of teams that lost or drew. Liu et al. ${ }^{[10]}$ reported that teams which are effective in dealing with aerial duels are more likely to dominate both the attacking and defending phases, eventually leading to a match win.

\section{Conclusion}

This study suggests that goalkeepers perform most match activities at a low intensity, with only a small proportion of actions executed at high-intensity. Goalkeepers of teams that lost covered significantly greater distances while sprinting compared to those of the teams that drew or won. The goalkeepers of teams that won had a higher number of ball recoveries than those of teams that lost and won. Therefore, the results of this study are important for soccer coaches in designing training programmes for goalkeepers so that they can meet the physical and technical demands of a game which could directly influence the competition's outcome.

Conflicts of interest and source of funding: The author declares that they have no conflict of interest and no source of funding.

\section{References}

1. Seaton M, Campos J. Distribution competence of a football clubs goalkeepers. Int J Perform Anal Sport 2011; 11(2):314324. [doi: 10.1080/24748668.2011.11868551]

2. White A, Hills SP, Cooke CB, et al. Match-play and performance test responses of soccer goalkeepers: A review of current literature. Sports Med 2018; 48(11): 2497-2516. [doi: 10.1007/s40279-018-0977-2] [PMID: 30144021]

3. Spalding J. Technical and physical match demands of a NCAA Division I soccer goalkeeper. Master's thesis. Paper 3176. East Tennessee State University, United States of America, 2017. https://dc.etsu.edu/etd/3176.

4. Liu H, Gómez MÁ, Lago-Peñas C. Match performance profiles of goalkeepers of elite football teams. Int J Sports Sci Coach 2015; 10(4): 669-682. [doi:10.1260/1747-9541.10.4.669] 
5. Kubayi A. Evaluation of match-running distances covered by soccer players during the UEFA EURO 2016. S Afr J Sports Med 2019; 31(1): 1-4. [doi: 10.17159/2078516x/2019/v31i1a6127]

6. Di Salvo V, Benito PJ, Calderón FJ, et al. Activity profile of elite goalkeepers during football match-play. J Sport Med Phys Fitness 2008; 48(4): 443-446. [PMID: 18997646]

7. Clemente FM, Couceiro MS, Martins FM, et al. Activity profiles of soccer players during the 2010 world cup. J Hum Kinet 2013; 38: 201-211. [doi:10.2478/hukin-2013-0060] [PMID24235995]

8. Rampinini E, Impellizzeri FM, Castagna C, et al. Technical performance during soccer matches of the Italian Serie A league: Effect of fatigue and competitive level. J Sci Med Sport 2009; 12(1): 227-233. [doi: 10.1016/j.jsams.2007.10.002] [PMID:18083631]

9. Dmitriy A, Mike N, Ilya V, et al. Validation and precision analysis of InStat Fitness system. InStat 2013; 1-14.

10. Liu H, Hopkins WG, Gómez MA. Modelling relationships between match events and match outcome in elite football.
Eur J Sport Sci 2016; 16(5): 516-525. [doi: 10.1080/17461391.2015.1042527] [PMID: 26190577]

11. Batterham AM, Hopkins WG. Making meaningful inferences about magnitudes. Int J Sports Physiol Perform 2006; 1(1): 50 57. [doi: 10.1123/ijspp.1.1.50 ] [PMID: 19114737]

12. Castellano J, Blanco-Villaseñor A, Alvarez D. Contextual variables and time-motion analysis in soccer. Int J Sports Med 2011; 32(6): 415-421. [doi.org/10.1055/s-0031-1271771] [PMID 21590641]

13. Bloomfield JR, Polman RC, O'Donoghue PG. Effects of scoreline on intensity of play in midfield and forward players in the FA Premier League. J Sports Sci 2005; 23(2): 192-193.

14. Kubayi A, Toriola A, Paul Y. The effects of ball-possession status on technical and physical indicators at the 2016 European Football Championship. Med Dello Sport 2018; 71(4): 533-539. [doi: 10.23736/50025-7826.18.03396-3]

15. Carling C, Williams AM, Reilly T. The handbook of soccer match analysis: A systematic approach to improving performance. London: Routledge, 2005, Pg 94. 